\title{
La prensa salvadoreña y la transparencia gubernamental: un instrumento para promover la cultura democrática de la información en la ciudadanía
}

\author{
Lya Ayala Arteaga \\ arteagapaz.israel@gmail.com
}

Egresada de Maestría en Comunicaciones de la Universidad Centroamericana "José Simeón Cañas", UCA-El Salvador

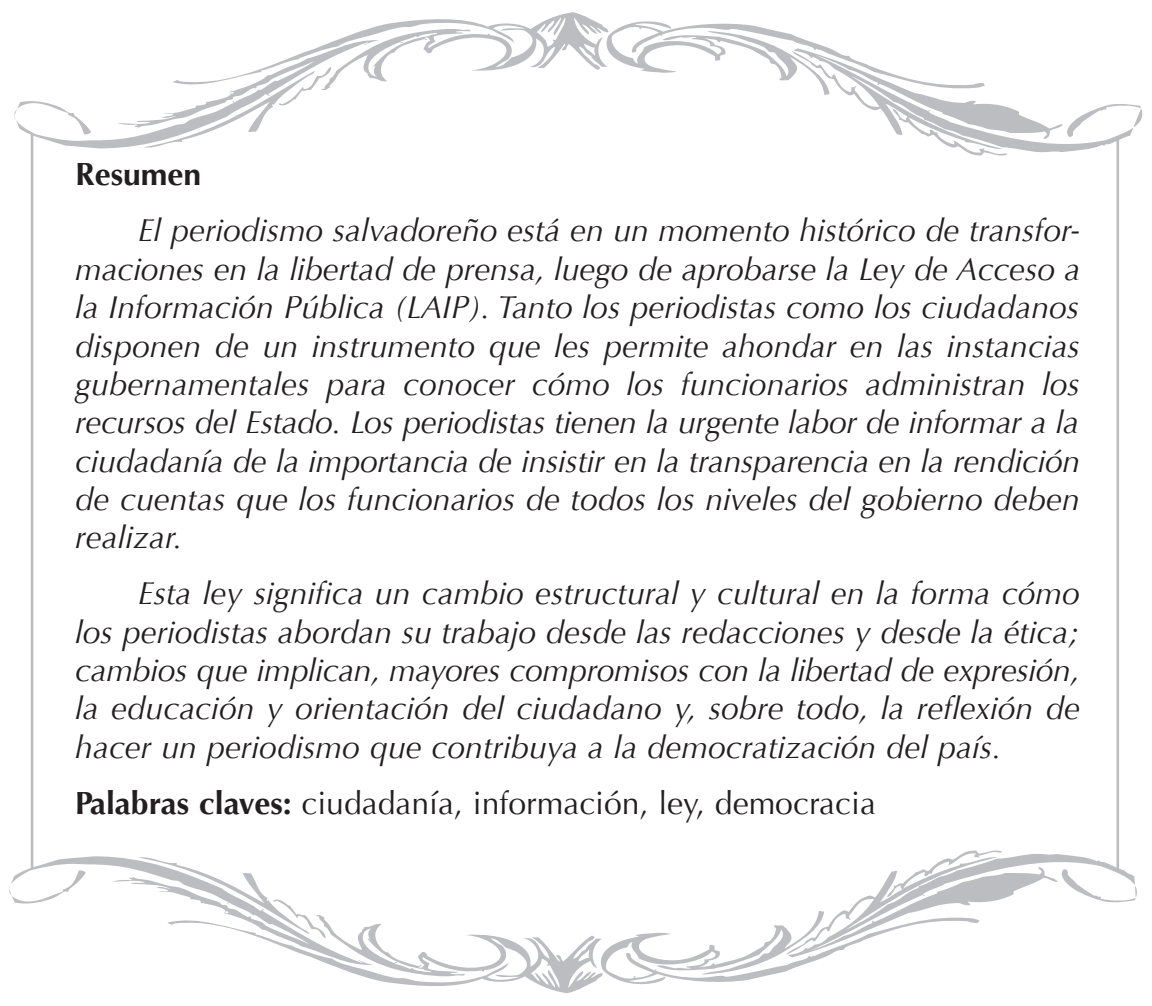




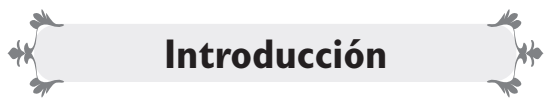

La aprobación de la Ley de Acceso a la Información Pública (LAIP) en 2011 significó un paso decisivo en la democratización de la información en El Salvador. Así la ciudadanía y los periodistas disponen de un instrumento que les posibilita pedir cuentas a los funcionarios y entidades gubernamentales sobre el uso que hacen de los recursos del Estado.

Más allá de este transcendental paso en la historia de la libertad de prensa y transparencia en la gestión pública salvadoreña; los periodistas tienen ahora el reto de ser partícipes, junto a la ciudadanía, de la transformación de una cultura de ocultamiento y corrupción en las administraciones públicas y dar paso a una cultura de la rendición de cuentas de los funcionarios a los ciudadanos.

En este sentido, son los periodistas los que deben propiciar las condiciones a través de trabajos de calidad periodística, una ética a prueba de sobornos y prebendas; amparados en la convicción de que el oficio de informar y comunicar construye los puentes, los caminos y las salidas hacia mejores condiciones para que los ciudadanos sean sujetos activos en exigir el derecho y acceso a la información pública.

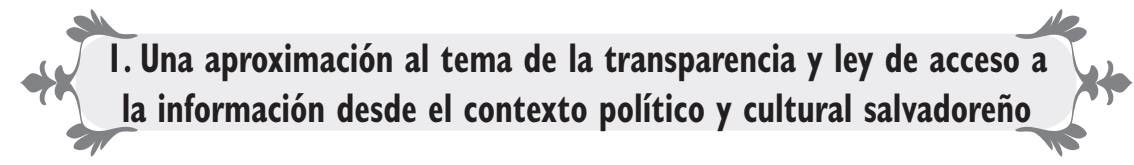

El Salvador transitó reciencómo administran los recursos del temente por las complejas calles que lo llevaron a la aprobación de una ley de acceso a la información pública (LAIP) en 2011, que fue impulsada por un grupo de ciudadanos: políticos, empresarios e intelectuales del país, con la premisa de que era necesario vincular el trabajo que las instituciones públicas realizan con la transparencia con que deben abordar su trabajo.

Otro aspecto importante fue que la ciudadanía tendría la posibilidad, amparada en la ley, de obtener información para conocer Estado los funcionarios de todas las carteras de gobierno, preguntar por qué no se cumplen reglamentos o se incumplen procedimientos en las instituciones del Estado que les atañen directamente; como salud, educación, seguridad, entre otros. Este valiosísimo instrumento les daría la posibilidad de ejercitar el derecho a esculcar en las profundidades de la cosa pública que les beneficia o les repercute en su vida como ciudadanos.

Así lo expresaban en la introducción del anteproyecto de ley de 
Acceso a la Información el grupo promotor de la iniciativa, auspiciados por la Fundación Salvadoreña para el Desarrollo Económico y Social (FUSADES) que lo impulsó desde 2008; señalaban en ese entonces, en un análisis sobre el valor político-cultural que tendría la aprobación de la ley, desde un lugar donde las manos del poder económico se mueven con bastante soltura, la relevancia de contar con una legislación que controlará a los funcionarios públicos.

Cosa interesante resulta que esta ley de transparencia y acceso a la información fuera la discusión imperativa entre los dueños de las empresas y políticos de derecha, durante un periodo político de cambios donde la derecha había dejado de ser la única que jugaba con los hilos del poder político y económico. Luego que la izquierda tomará las riendas de la administración pública, la ley no tuvo tanta presencia en las promesas políticas del partido de izquierda ya entronizado, estos cambios históricos iniciados hace cuatro años son importantes porque le dan viabilidad a los procesos democráticos de alternar la administración de la cosa pública e impulsar desde estas nuevas elecciones un Estado más saludable comprometido y reflexivo del uso que los gobernantes hacen de los bienes públicos.

¿Cómo enfrenta El Salvador estas transformaciones políticas y culturales, después de una guerra civil; donde casi corriendo, la historia lo lleva a aprobar una ley de acceso a la información pública, una de las pocas que hay en América Latina?

Por supuesto, con dificultades que son necesarias para ir legitimando el proceso, puesto que todos los involucrados tiene que reflexionar sobre la urgencia de controlar a los que tienen el poder de tomar decisiones relevantes para el desarrollo de la ciudadanía salvadoreña; por eso resulta casi una obviedad que el grupo promotor fuera el de los empresarios y académicos, ambos con intereses distintos apoyaron e impulsaron lo que sería uno de los instrumentos más valiosos para que la cultura de observación de los ciudadanos formará parte de la incipiente libertad de prensa, para que haya responsabilidad desde todos los sectores y el proceso democrático se vaya consolidando en la cultura salvadoreña, que los ciudadanos puedan y deban pedir cuentas a los funcionarios y mandatarios y exijan transparencia en el manejo de los bienes públicos (Umaña, 2010).

Veamos a continuación el papel que juega la prensa salvadoreña, sobradamente conocida como un aliado de los grupos de poder, y la forma en que aborda el tema de la transparencia en las instancias gubernamentales, lugares intocables hace una década, y que ahora el 
mismo FUSADES apela a que se haga público todo movimiento en sus pasillos.

La prensa salvadoreña ha jugado un papel de gran relevancia en la puesta en escena, a través de su agenda mediática, de temas controversiales de políticos, empresarios e instituciones; pero de manera fragmentada y limitada en los contenidos. Así que se ha quedado corta en la manera en que presenta la realidad por la debilidad que hay en el concepto histórico de periodismo de investigación, que para la profesión en el país se resume a una entrada tardía en la academia, las primeras escuelas de periodismo inician su inscripción en el Ministerio de Educación (MINED) hasta los 90`s, exceptuando a la Universidad de El Salvador (UES) que lo había hecho en los 70’s.

La carrera hacia el periodismo profesional ha tenido y tiene escollos graves que superar: la guerra civil y la cobertura sesgada y manipulada de la información hacia afuera del territorio dejó como producto un abordaje limitado e ideologizado del periodismo hasta nuestros días que sigue sugestionando la visión, tanto de los periodistas como de los consumidores de información, de la realidad nacional: siempre se sospecha que hay gato encerrado y de esa manera se construyen los formatos, se redacta con poco astucia periodística, limitado análisis y dudosas fuentes oficiales.
También la lenta profesionalización y la instauración de la academia en las universidades y la escasa capacitación de los periodistas en los medios de comunicación ha imposibilitado un debate integral sobre las falencias de la prensa nacional; otro elemento es la intromisión de grupos sociales civiles, medios de producción y el gran capital (empresas, transnacionales, sectores económicos y gremiales) que manejan desde adentro la agenda mediática y la relación de los periodistas con los políticos de turno que les ofrecen primicias a periodistas mal intencionados o novatos que publican esta información ya manipulada y con un fin específico de mantener a salvo actos de corrupción y abuso de poder.

Estos factores colocan la situación actual del periodismo nacional en una encrucijada respecto al tema de la transparencia y para llegar hasta allí, la prensa salvadoreña ha tenido y tiene que bregar con el mismo poder político y económico que otrora manejaba completamente la agenda mediática a través de grandes inversiones en publicidad en los medios impresos y televisión. Aunque sigue siendo de esta manera, los medios electrónicos y las redes sociales han sido una punta de lanza que modificó la agenda de los principales periódicos y noticiarios del país. 
Un ejemplo de ello es que hace dos años, en 2011, en una capacitación de la Asociación de Periodistas de El Salvador, que trataba el tema del periodismo de investigación, un colega de un noticiario de televisión narró el caso de un reportaje que hablaba sobre la contaminación que una empresa local estaba produciendo no sólo al medio ambiente, sino a quienes trabajaban en ella. Se habían documentado casos de personas que habían adquirido cierta enfermedad e inclusive de fallecidas como producto de la contaminación.

El noticiario anunció horas antes la publicación del reportaje para su espacio de la noche. Sin embargo, en horas de la mañana los propietarios de esa empresa se presentaron, hablaron con el director y por la noche, después de haberlo publicitado grandemente, éste no se publicó. No hubo explicaciones, los televidentes quedaron burlados y las llamadas telefónicas para acusar al periodista de «vendido» no se hicieron esperar. El periodismo quedó por los suelos. (Herrera Palacios, 2000)

A pesar de las dificultades, las nuevas Tecnologías de la Información y Comunicación (TIC) han traído una nueva visión y recursos para exponer los temas que antes era complejo informar a la ciudadanía. En El Salvador las TIC y las redes sociales tiene una relevancia en el desarrollo de un periodismo de investigación más eficiente que toca las fibras de las instancias gubernamentales y las acciones de los gobernantes. La ciudadanía se ha convertido en lo que algunos Ilaman "periodista ciudadano", cuando lo adecuado sería considerar esta responsabilidad ciudadana de la denunciar, como parte de la cultura democrática de la libertad de expresión.

Algunos de los medios digitales que abordan con profundidad la agenda gubernamental, la empresarial y de otros sectores son los periódicos digitales El Faro, Contrapunto, Voces y actualmente el $\mathrm{Blog}$, aunque algunos lo sostienen organizaciones no gubernamentales con tendencia hacia la izquierda o financiados por organismos como la Agencia de los Estados Unidos para el Desarrollo Internacional (USAID, por sus siglas en inglés), han podido seguirle la pista a temas sobre corrupción y ha expuesto abusos de grandes empresas y transnacionales con el tema de la explotación minera, contaminación ambiental de los mantos acuíferos, corrupción dentro de la Asamblea Legislativa, Corte de Cuentas y hasta la forma en que el mismo mandatario salvadoreño utiliza la partida secreta. Estos temas medulares en la cultura política, cultural y económica del país eran impensable en la agenda periodística hace una década.

El periodismo salvadoreña evidentemente ha avanzado en los 
temas de profesionalización, existen en las universidades las carreras de periodismo y comunicación social, hay periodistas con formación técnica y teórica. Ahora el punto es cómo se produce ese retroceso cuando llegan a las redacciones. Veamos lo que señala el doctor en ciencias de la comunicación, Antonio Herrera Palacios, sobre este punto.

"No todo es color de rosa. Al inicio de este artículo, he citado el texto de una caricatura que ilustra la conversación entre un director de periódico y un reportero. La comparación es simple: el periodista puede investigar, pero siempre y cuando no se lesionen los intereses del medio. El principal problema radica allí, en el medio mismo, en su estructura y en su forma de concebir el ejercicio periodístico, no tanto en quienes ejercen directamente -es decir los reporteros- sino en quienes les dirigen. Aún no se ha cambiado la mentalidad de que cuando se tocan los intereses comerciales del medio, la labor social del periodismo queda relegada a un segundo plano. Temas que menoscaben la integridad o imagen de una empresa que es potencialmente un cliente que deja muchos ingresos para el medio, son prácticamente intocables" (Herrera Palacios, 2000).

Así se explica, en parte, que muchos periodistas con una base sólida en la profesión terminen huyendo hacia otras áreas de la comunicación y la publicidad y se desvinculen de una tarea valiosa para el desarrollo de la búsqueda de información y se queden los periodistas que estarán al servicio de determinados intereses, que minan el proceso necesario para esculcar en las oficinas públicas y en los pasillos por donde circulan los políticos y las decisiones que deben ser mostradas a la ciudadanía.

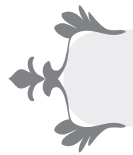

\section{Poder político, poder económico y la ciudadanía} ¿Para quién trabaja la prensa?

Uno de los factores que apuntaremos aquí es la fuente de donde obtienen los periodistas la información para elaborar su trabajo y será la fuente el tema de reflexión de este apartado, cómo y dónde buscan los periodistas la "carnita" para escribir este o aquel tema que a la ciudadanía le está costando grandes cantidades de impuestos y no "mira" en la realidad lo que se hace con estos dineros.

El periodista acude inevitablemente a la fuente oficial, seguramente a un administrador o gerente de segundo nivel que le explica con algunos documentos ininteligibles (elaborados por técnicos) la labor que se hace; luego, acude a un 
par de ciudadanos y toma declaraciones, finalmente unas fotos $y$ hasta allí...

Sin embargo, explorar la fuente, abrirle surcos al tema hasta redondearlo y colocarlo en contexto es otra cosa: el periodista tiene que ir a la fuente principal si es posible, el ministro, el director de la dependencia, es decir, a quien le puede dar una respuesta responsable de lo que se está haciendo, a partir de allí ya puede elaborar y exponer en su trabajo datos e información que proponga a la ciudanía reflexión y cuestionamiento de lo que se hace con los fondos públicos.

Esa sería la imagen cuasi ideal del trabajo periodístico y su relación con la transparencia en las entidades públicas y gubernamentales, pero cómo llegar hasta allí y por qué, Guerrero (2008) señala en una democracia los medios tienen dos funciones: "la libertad de expresión y de prensa en una democracia. Por una parte, se trata de una función informativa: la libre expresión permite el flujo de información necesaria; por la otra, se trata de una función crítica: los medios pueden servir de guardián en favor de los ciudadanos y ayudar asimismo a la libre crítica en el ejercicio del poder, y a la evaluación del desempeño de los políticos y de las instituciones.". Ya situados en las funciones que deben realizar estamos claros que su trabajo debe estar enfocado en la información y la crítica para que la ciudadanía observe y participe activamente en la forma que se administran los recursos del Estado, por lo que debe disponer de información veraz (Vergara, 2008).

Así, los medios pueden muy bien quedarse con la imagen de ser los que descubren los desmandes o abusos de los gobernantes, pero más allá de eso, el periodismo salvadoreño debe fundamentar las preguntas que hace a las fuentes: el periodista salvadoreño debe conocer por qué pregunta y luego cómo lo contextualizará y este ejercicio de alguna manera lo llevará a cuestionar con profundidad las limitaciones del sistema democrático incipiente del país y a comprender que el uso del poder por un sector se remonta a la misma historia salvadoreña o como explica Vergara: que en la tradición liberal la autoridad oprime los derechos y que, por lo tanto, los medios de comunicación se vuelven instrumentos de defensa "como aliados naturales de los ciudadanos", preservando su libertad del poder político. 


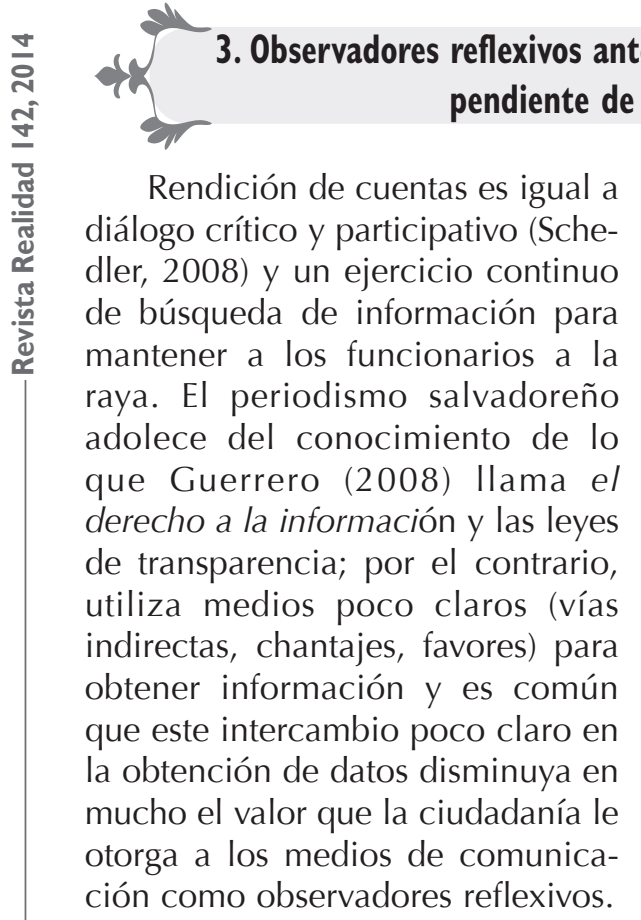

La labor del periodista deberían ser solicitar, por las vías legales que ya existen, la rendición de cuentas de las instancias públicas para controlar el poder político a través de esta información; no es para justificar ni sancionar el trabajo de los funcionarios públicos, es decir, no eliminar las actividades en las que se administra la cosa públicas, no, es que la rendición de cuentas lo que hará es monitorear que los proceso se cumplan bajo la ley (Schedler, 2008).

El periodismo nacional no debe buscar sólo información para exponer a los funcionarios para ridiculizarlos, sino que los funcionarios digan qué hacen con los recursos del Estado y que asuman la responsabilidad de hacerlo de la mejor manera, con transparencia, y ofrecer desde una perspectiva de compromiso con la libertad de expresión y la libertad de prensa su posición con respecto al trabajo que realiza, esto incentivaría el derecho a la información y participación en la democratización del país, donde la calidad de la democracia se logra cuando los ciudadanos equilibran los niveles de información (Levine Molina, 2007) y los medios de comunicación están dispuestos a explorar que hay en las carteras de los funcionarios públicos.

Y sean los medios de comunicación salvadoreños los que contribuyan a que la ciudadanía conozca cómo los contrapesos de poder se van manejando, van surgiendo las sanciones hacia los que están en puestos públicos, los que tienen las riendas de las decisiones. No es que el periodismo deba tomarse la batuta del poder absoluto y sea un árbitro entre los gobernantes y funcionarios y la ciudadanía, sino que debe abrir las posibilidades de información a los ciudadanos para que estos tengan un abanico de propuestas para que puedan decidir quién será el que deba administrar el Estado, que sepan y conozcan que disponen de un sistema de información libre que les permite saber cómo se administran sus impuestos. 
Esa debería ser la labor del periodismo salvadoreño en estas actuales circunstancias en que la ley de acceso a la información empieza a tomar relevancia entre los mismos periodistas y los ciudadanos; cuando se eligen funcionarios de primer nivel, se aprueban presupuestos, se acercan las elecciones. Un ejemplo claro, pero minimizado y poco analizado por los mismos medios de comunicación, es el avance de la participación ciudadana en la toma de decisiones en las elecciones pasadas cuando pidió cuentas al gobierno de derecha que había tenido por 20 años el gobierno, Alianza Republicana Nacionalista ARENA, y al no obtener respuestas claras, es decir, en obras palpables; corrupción abiertamente conocida y demostrada, no votó por ellos.

La ciudadanía entonces le cedió al partido combinado de centro izquierda Frente Farabundo Martí para la Liberación Nacional (FMLN) y un grupo de empresarios e intelectuales aglutinados en "los amigos de Mauricio" la administración del gobierno. La ciudadanía ha pedido las cuentas correspondientes en este periodo de cuatro años, a escaso un año de finalizar el periodo de este combinado político, por este periodo, porque están más conscientes del valor de las elecciones, más atentos a las acciones de los funcionarios; los medios alterativos electrónicos y las redes sociales han tenido mucho que ver en incentivar a la población a observar atentos cómo se administran los recursos y, hay que poner suma atención a este fenómeno, la ciudadanía denuncia y opina acerca de las actuaciones de los funcionarios.

El periodista debe estar atento a estos cambios culturales y sociales de la ciudadanía, a estas señales que la ciudadanía ofrece para que con la libertad de expresión se defiendan la libertad de los ciudadanos de comunicarse y expresar su punto de vista sin temor a las represalias, los periodistas amparados en medios de comunicación responsables y comprometidos con el desarrollo social, cultural e histórico del país deben investigar, buscar y analizar sobre la realidad nacional (Guerrero, 2008).

El binomio medios de comunicación y ciudadanía que cuenta con herramientas como la Ley de Acceso a la Información Pública procede de manera más abierto e investigativa, logra efectos saludables para aprender a vivir democráticamente, veamos como la ley y la continua aplicación de los conceptos de la transparencia como balanza en los ejercicios del poder político, agiliza la participación de ciudadanos y ciudadanas en este proceso democrático. 


\section{El atropellado inicio de la ley de acceso a la información pública}

Según la Asociación de Periodistas de El Salvador (APES) el país se ubica en la posición 38 en la clasificación Mundial de la Libertad de Prensa que Reporteros sin Fronteras (RSF) realizó en 2012. Posición "envidiable" es el calificativo que el sitio web de transparencia activa http://www.transparenciaactiva.gob. sv utiliza en su titular.

La existencia misma del sitio apuntala una serie de aplicaciones importantes que la LAIP señala. Es verdad que se ha corrido, como dijimos al principio de este escrito, con la aprobación de la ley con el tira y encoge de los poderes y contrapesos de poderes de los empresarios, políticos y dueños de los medios de comunicación; pero el resultado de este pulso ha sido aprobar una ley que posibilita a los ciudadanos poder conocer la gestión pública que realizan los funcionarios.

A pesar del avance significativo de la aprobación y puesta en marcha de las herramientas y procesos de la ley, algunos periodistas ven con poco optimismo la aplicación de ésta. En la misma nota emitida por transparencia activa, el presidente de APES, José Luis Benítez, señala que aunque la realidad de la prensa no es óptima ni ideal, si está bastante mejor debido a las condiciones políticas de escasa represión y persecución a la prensa.

Los optimismos fluyen recién iniciada la aplicación de la ley, pero en El Salvador la cultura de esconder los trapos sucios de los políticos todavía sigue siendo una madeja difícil de desenredar, el autoritarismo todavía es el pan de cada día entre los periodistas y los funcionarios de todos los niveles; una noticia sospechosa para los ciudadanos que poco a poco van conociendo de qué trata la ley y qué pueden hacer y solicitar a las oficinas de la LAIP que deben estar en cada ministerio u oficina pública. Los ciudadanos deben ahora acostumbrarse y ejercer este derecho que les otorga la ley. Y el periodismo salvadoreño debe, por su parte, informar y educar urgentemente sobre el tema.

No es para menos, en el primer monitoreo al cumplimiento de la LAIP de la ONG Iniciativa Social para la Democracia (ISD) señala que luego de la aprobación de la ley hubo un retraso de casi un año para poner en funcionamiento el Instituto de Acceso a la Información Pública (IAIP), institución clave para dar paso a la acciones, nombrar funcionarios y establecer las Unidades de Acceso a la Información Pública (UAIP).

Fue la misma APES y algunos pocos periodistas los que insistieron 
en cumplir los plazos para que la ley contara con todos los recursos para empezar a funcionar, y ese compromiso de velar por la libertad de prensa se ha quedado en pocas voces.

La tarea es ardua para los periodistas, quienes tienen la labor urgente de tomar presencia y compromiso con los procesos democráticos haciendo uso del instrumento $y$, sobre todo, generar estos cambios desde las redacciones donde los dueños de los medios de comunicación todavía se resisten a dar derecho de respuesta a cubrir diversidad de fuentes a mostrar mayor equilibrio en la distribución de la agenda en los temas políticos y sociales del país.

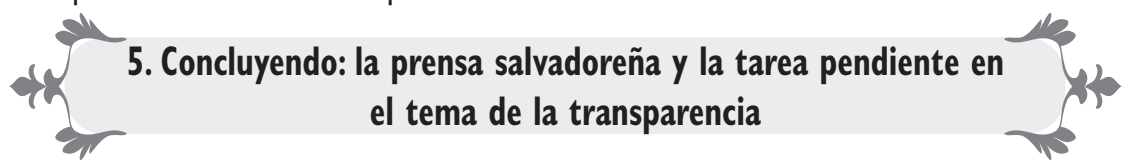

La prensa salvadoreña debe cumplir su papel en la historia, no para ser un nodo de poder, sino un medio para transformar la cultura de corrupción y abuso de poder a través de la exposición de la agenda del gobierno y las instituciones públicas a la ciudadanía, el trabajo de las intuiciones públicas, funcionarios y gobernantes es necesario que sea conocido y evaluado por la ciudadanía.

Para ello la prensa necesita profesionalizarse y desvincularse del poder para fiscalizar al gobierno, saber por qué la transparencia es un modo de afianzar la democracia, involucrarse en la agenda pública y mediática con compromiso para convertirse en "un interlocutor privilegiado que pueda influir en la cantidad y calidad de información que la gente recibe, así como la forma en que se presenta tal información. Por tanto, los medios de comunicación, públicos como privados, están obligados a mantener un equilibrio entre su derecho a la libertad de prensa y una base mínima de criterios de responsabilidad informativa ante sus lectores y audiencias" (Guerrero, 2008).

No serán sólo los periodistas, ya que inevitablemente son empleados de una empresa o responden a un grupo, no serán sólo los políticos ni los que impulsan las leyes sobre la libertad de expresión y de prensa, tampoco los empresarios o los mismos políticos. Serán todos: los ciudadanos y los actores sociales que cuentan con las herramientas y mecanismos necesarios irán aprendiendo a convivir de otra manera, en este sentido, es que la los medios de comunicación cumplen ese papel de gran relevancia de fiscalizar de promover de generar reflexión y de proponer cambios y transformaciones. 
La prensa no es el cuarto poder, no lo es, sólo es una parte del engranaje que mueve a las sociedades, pero un engranaje que puede impulsar cambios y transformaciones en la cultura de los ciudadanos e impulsar y apoyar a consolidar la democracia.

\section{Referencias bibliográficas}

os Guerrero Manuel Alejandro (2008). Medios de comunicación y la función de transparencia, Instituto Federal de Acceso a la Información Pública (IFAI), Cuaderno de transparencia 03, pp 18-32- 36.

os Levive H Daniel, Molina Enrique José (2007) La calidad de la democracia en América Latina: una visión comparada, América Latina Hoy, abril, número 045, Universidad de Salamanca, Salamanca, España, pp. 22-23.

os Schedler Andreas (2008) ¿Qué es la rendición de cuentas?, Instituto Federal de Acceso a la Información Pública (IFAI), Cuaderno de transparencia 03.

os Vergara Rodolfo (2008) La Transparencia como problema, Instituto Federal de Acceso a la Información Pública (IFAI), Cuaderno de transparencia 05.

\section{Bibliografía WEB}

os Palacios Antonio Herrera (2000) Universidad Tecnológica de El Salvador, recuperado de http://www.revistaprobidad.info/007/art07.html

os Alvarado Luis (2012), Dos declaraciones sobre la transparencia en El Salvador, recuperado dehttp://www.transparenciaactiva.gob.sv/laip/2012/ dos-declaraciones-sobre-la-trasparencia-en-el-salvador/

os Grupo promotor FUSADES (2010) Ley de transparencia y acceso a la información pública: una ley desde la sociedad civil salvadoreña, recuperado de http://www.accesoinformacionelsalvador.org/documentos/ documentogp.pdf

os Libertad de prensa en El Salvador está en una posición "envidiable", recuperado de http://www.transparenciaactiva.gob.sv/libertad-de-prensaen-el-salvador-esta-en-una-posicion-envidiable/\#sthash.wYRthIDo.dpuf

os Ley de Acceso a la Información Pública (LAIP), recuperado de http:// www.cnr.gob.sv/index.php?option=com_phocadownload\&view=categ ory\&id=35:ley-de-acceso-a

os Iniciativa Social para la Democracia (ISD), recuperado de http://isd. org.sv/wp/wp-content/uploads/2012/11/PRIMER-MONITOREO-ALCUMPLIMIENTO-DE-LA-LEY-DE-ACCESO-A-LA-INFORMACI\%C3\%93NP\%C3\%9ABLICA.pdf 